\title{
Impaired Emotional Mirroring in Parkinson's Disease-A Study on Brain Activation during Processing of Facial Expressions
}

\begin{abstract}
Anna Pohl ${ }^{1,2 *}$, Silke Anders ${ }^{3}$, Hong Chen ${ }^{2}$, Harshal Jayeshkumar Patel ${ }^{2,5}$, Julia Heller, Kathrin Reetz ${ }^{4,6}$, Klaus Mathiak ${ }^{6,7}$ and Ferdinand Binkofski ${ }^{2,5,6}$

${ }^{1}$ Department of Psychology, University of Cologne, Cologne, Germany, ${ }^{2}$ Division of Clinical Cognitive Sciences, RWTH Aachen University, Aachen, Germany, ${ }^{3}$ Department of Neurology, University of Lübeck, Lübeck, Germany, ${ }^{4}$ Department of Neurology, RWTH Aachen University, Aachen, Germany, ${ }^{5}$ Institute of Neuroscience and Medicine (INM-4), Research Center Jülich GmbH, Jülich, Germany, ${ }^{6}$ Jülich Aachen Research Alliance (JARA), Translational Brain Medicine, Aachen, Germany, ${ }^{7}$ Department of Psychiatry, Psychotherapy, and Psychosomatics, RWTH Aachen University, Aachen, Germany
\end{abstract}

Background: Affective dysfunctions are common in patients with Parkinson's disease, but the underlying neurobiological deviations have rarely been examined. Parkinson's disease is characterized by a loss of dopamine neurons in the substantia nigra resulting in impairment of motor and non-motor basal ganglia-cortical loops. Concerning emotional deficits, some studies provide evidence for altered brain processing in limbic- and

OPEN ACCESS

Edited by:

Oscar Arias-Carrión, Hospital General Dr. Manuel Gea Gonzalez, Mexico

Reviewed by:

Matteo Bologna,

Sapienza Università di Roma, Italy

Silmar Teixeira

Federal University of Piauí, Brazil

*Correspondence:

Anna Poh

anna.pohıuni-koeln.de

Specialty section: This article was submitted to Movement Disorders, a section of the journal Frontiers in Neurology

Received: 22 September 2017 Accepted: 29 November 2017 Published: 18 December 2017

Citation:

Pohl A, Anders S, Chen H, Patel HJ, Heller J, Reetz K, Mathiak K and Binkofski F (2017) Impaired Emotional Mirroring in Parkinson's DiseaseA Study on Brain Activation during Processing of Facial Expressions.

Front. Neurol. 8:682. doi: 10.3389/fneur.2017.00682 lateral-orbitofrontal gating loops. In a second line of evidence, human premotor and inferior parietal homologs of mirror neuron areas were involved in processing and understanding of emotional facial expressions. We examined deviations in brain activation during processing of facial expressions in patients and related these to emotion recognition accuracy.

Methods: 13 patients and 13 healthy controls underwent an emotion recognition task and a functional magnetic resonance imaging (fMRI) measurement. In the Emotion Hexagon test, participants were presented with blends of two emotions and had to indicate which emotion best described the presented picture. Blended pictures with three levels of difficulty were included. During fMRI scanning, participants observed video clips depicting emotional, non-emotional, and neutral facial expressions or were asked to produce these facial expressions themselves.

Results: Patients performed slightly worse in the emotion recognition task, but only when judging the most ambiguous facial expressions. Both groups activated inferior frontal and anterior inferior parietal homologs of mirror neuron areas during observation and execution of the emotional facial expressions. During observation, responses in the pars opercularis of the right inferior frontal gyrus, in the bilateral inferior parietal lobule and in the bilateral supplementary motor cortex were decreased in patients. Furthermore, in patients, activation of the right anterior inferior parietal lobule was positively related to accuracy in the emotion recognition task.

Conclusion: Our data provide evidence for a contribution of human homologs of monkey mirror areas to the emotion recognition deficit in Parkinson's disease.

Keywords: neurodegeneration, mirror neurons, emotion, functional MRI, facial emotions, Parkinson's disease 


\section{INTRODUCTION}

Patients with idiopathic Parkinson's disease (PD) suffer from a wide range of emotional disturbances including subjective feeling of emotions and the related physiological arousal states, but also recognizing and expressing them (1). These deficits are important as life quality is reduced and social interactions are hampered. Already, an early study suggested a negative influence of impaired facial expressiveness on interpersonal relationships (2), and psychosocial functioning was considered a key factor for health-related quality of life in PD [e.g., Ref. (3)]. Dopamine deficiency due to loss of nerve cells in the substantia nigra results in an imbalance of dopaminergic innervation in subcortico-cortical circuits, which causes typical motor and non-motor symptoms in PD (4). In these circuits, cortical and limbic areas are connected to different parts of the striatum, globus pallidus, substantia nigra, and thalamus (5).

Up to now, studies investigating emotion processing in $\mathrm{PD}$ have mostly focused on the orbitofrontal cortex (OFC) and the amygdala. A meta-analysis of structural imaging data revealed a reduction of left lateral OFC (BA47) gray matter volume in PD (6), which in turn was associated with disturbed facial emotion recognition (7). Furthermore, OFC activation has been found to be reduced in PD [left hemisphere (8), right hemisphere (9)] and in asymptomatic Parkin mutation carriers [left hemisphere (10)] during processing of affective facial expressions. It was assumed that diminished activation of the OFC is part of a dysregulation of the "mesolimbic gating loop" or the "lateral-orbitofrontal gating loop" (10). Pathological changes of the amygdala in PD involve the presence of Lewy bodies $(11,12)$, volume loss $(7,12)$, and a reduction of the dopamine binding level (13). Moreover, bilateral amygdala activation was found to be reduced in PD during an emotion discrimination task (14).

Another line of evidence supports the hypothesis that the link between emotion recognition deficits and facial motor impairments is closer than previously assumed. Reduced facial expressiveness is an important clinical symptom of $\mathrm{PD}$, which might be useful for clinical evaluation of PD in the future [for a review, see Ref. (15)]. Three studies showed a significant relation voluntary control of facial muscles and of emotion recognition deficits (16-18). Response latencies during emotion recognition were shown to be negatively correlated with the amplitude of facial muscle responses in PD (19). Moreover, a relation between emotion recognition and a lack of automatic mimicry during observation of emotional facial expressions was recently reported (20). Although one study did not find a significant relation between velocity and amplitude of voluntary facial muscle activation and emotion recognition [note that sample size was small and effect size was not reported (21)], we assume that further examination of the association between emotion recognition deficits and facial motor impairments are valuable. Based on current theories of social cognition, it was assumed that the "neural resonance" in the observer's motor system that normally facilitates understanding of facial expressions (22) is disturbed in PD (20). These theories are based on the detection of a special class of visuomotor neurons in inferior frontal area F5 [for a review, see Ref. (23)] and anterior inferior parietal area
PFG of the macaque monkey (24-26). Area F5 is supposed to be the homolog of the human pars opercularis of the inferior frontal gyrus (IFGop) (27), area PFG the homolog of the human cytoarchitectonic area PFt, which is located in the supramarginal gyrus (28). Both areas are anatomically connected (29). The so-called "mirror neurons" were found to be activated not only during action execution but also during mere observation of goal directed actions and are thought to be a prerequisite for action understanding (30). It was assumed that observed facial expressions are transformed into motor and/or somatosensory representations, respectively, which help to understand the emotion (22). Overlapping activation during production or imitation and observation of emotional facial expressions was taken as evidence for the relevance of mirror neurons in the human IFGop and the anterior inferior parietal lobule (aIPL) for emotion processing $(22,31-34)$.

Up to now, the relation of emotion recognition and motor impairment in $\mathrm{PD}$ was examined in behavioral studies only. Here, we aimed at studying the neural substrate of these related disturbances. In a previous study, we showed that activation of right IFGop during execution and observation of pleasant facial expressions is increased in subclinical carriers of a single mutant Parkin allele. Importantly, increased activation in the IFGop was positively related to performance in an emotion recognition task. The Parkin allele mutation is a cause for early onset PD (35). It is accompanied by a reduction of the 18F-fluoro-L-DOPA metabolism (36), but mutation carriers do not suffer from clinical motor symptoms. It was assumed that the dopamine reduction is compensated by the motor system (37). In line with this, increased activation of the IFG related to improved emotion recognition was assumed to be compensatory (10). Based on this study, we examined whether patients with manifest idiopathic Parkinson's disease show altered emotion recognition abilities and altered involvement of human homologs of mirror neuron areas and/or structures embedded in limbic- or lateral-orbitofrontal-basal ganglia circuits during execution and observation of emotional facial expressions. Impaired emotion recognition was postulated for the patient group. We mainly focused on activation of the right IFGop in manifest PD and assumed decreased activation due to a breakdown of the compensatory mechanism present in the subclinical stage in individuals with a Parkinson's diseaseassociated mutation (10). Exploratory, we were also interested in potential activation differences in (i) bilateral aIPL, (ii) bilateral amygdala, and (iii) left OFC. Finally, we asked if altered brain activation was related to emotion recognition abilities in PD.

\section{MATERIALS AND METHODS}

\section{Procedure and Participants}

Patients were recruited from the Movement Disorders Outpatient Clinic at the Department of Neurology and control participants were recruited in Aachen and the surrounding area. Before inclusion in the study, patients and volunteers were screened for mental disorders using a short German version of a DSM-IV interview [SKID-PIT light (38)]. Only volunteers 
with no self-reported history of mental illness were included in the study. 14 patients with Parkinson' disease (PD) and 13 healthy controls (HC) underwent the study protocol. Data of one patient had to be excluded from analysis due to technical problems. Data of $13 \mathrm{PD}(5 \mathrm{f}, 8 \mathrm{~m})$ and 13 age-matched HC with no self-reported history of neurological diseases $(6 \mathrm{f}, 7 \mathrm{~m})$ were analyzed. All participants were right handed (39) and had normal or corrected-to-normal vision. PD were medicated with antiparkinsonian drugs according to their symptoms and measured in the "ON" state. The mean levodopa equivalent daily dose (LEDD) [calculated according to Ref. (40)] across all PD was $586.52(S D=494.88)$. The averaged disease duration for PD was 5.94 years $(S D=4.39$, range $0.9-15.4$ years). Patients with PD underwent the Mini-Mental-Status-Test (41). The averaged sum score was $28.31(S D=1.55 ; \operatorname{Min}=25)$. An experienced movement disorder specialist assessed motor dysfunction in PD using the motor subscale of the Unified Parkinson's Disease Rating Scale [(UPDRS-III) (42) $M=24.21(N=12, \mathrm{SD}=9.60)$ ].

Groups did not differ with regard to age [Median $(M d n)_{\mathrm{HC}}=65$, $\left.M d n_{\mathrm{PD}}=68 ; U=-0.13 p=0.898\right]$, years of school education $\left[M_{\mathrm{HC}}=10.92, \mathrm{SD}_{\mathrm{HC}}=1.32, M_{\mathrm{PD}}=10.15, \mathrm{SD}_{\mathrm{PD}}=1.68 ; t(24)=1.3\right.$, $p=0.206$ ] or crystallized intelligence, assessed by a German neuropsychological test [Mehrfachwahl-Wortschatztest version B MWT-B (43) $M_{\mathrm{HC}}=118.62, S D_{\mathrm{HC}}=17.15, M_{\mathrm{PD}}=113.54$, $\left.S D_{\mathrm{PD}}=20.17 ; t(24)=0.69, p=0.496\right]$.

\section{Experimental Setup}

Participants underwent neuropsychological assessment and a functional magnetic resonance imaging (fMRI) measurement, completed a post-scanning questionnaire (PSQ) and a computerbased emotion recognition test.

We used the same experimental setup as in our study examining subclinical carriers of a single mutant Parkin allele (10) that was based on an earlier study on healthy participants (34). To assess emotion recognition, we used the Facial Expressions of Emotions-Stimuli and Test battery [FEEST (44)]. The FEEST contains two tests comprising photographs and morphed photographs of emotional facial expressions from the Pictures of Facial Affect series (45). These photographs are commonly used to estimate emotion recognition deficits in PD (46). In our study, participants underwent the Emotion Hexagon test. In this test, photographs of two emotions are blended, whereby the proportion of each emotion differs. Since sensing and expressing mixtures of emotions is very common (47), we assume a high ecological validity of the Emotion Hexagon test. The fMRI experiment enabled us to examine disturbances of the involvement of human homologs of mirror neuron areas in PD.

\section{Emotion Hexagon Test}

Participants were asked to judge emotions in a six-alternative forced choice paradigm from morphed emotional facial expressions of different levels of difficulty (44). The FEEST consists of blended continua of two different emotional facial gestures, which are presented on a computer screen (45). Similar emotional expressions (happiness-surprise, surprise-fear, fearsadness, sadness-disgust, disgust-anger, anger-happiness) are blended with different proportions of each emotion (90-10,
$70-30,50-50,30-70,10-90 \%)$ resulting in a total number of 30 morphs. The test contains 150 test trials split into five runs, in which, the 30 morphs were presented once in a randomized order. In total, each emotion occurred ten times in each degree of difficulty. The labels of the six basic emotions were provided at the bottom of the computer screen as alternative choice. Participants were asked to name the emotion that best described the facial expression displayed on the computer screen. Participants were familiarized to the task by 30 test trials prior to the actual test. Responses were entered into the computer by the experimenter. Responses to the 90-10 and the 70-30\% morphs were defined correct if the predominant emotion was chosen by the participant. Responses to the 50-50\% morphs were considered correct if one of the two emotions included in the morph was chosen by the participant.

\section{fMRI Experiment and Post-Scanning Rating Procedure}

The stimulus material consisted of video clips, which had previously been used by us and our collaboration partners (10, 34, $48,49)$. These clips consisted of emotional (smile), nonemotional (lip protrusion), and neutral stimuli (relaxed face without motion). The latter was used as a high level baseline. Each of the three facial expressions was shown by 24 actors ( $12 \mathrm{men}$ ), giving a total of 72 videos. Each video started with the actor displaying a neutral face for $1 \mathrm{~s}$ than expressing the facial gesture for $3 \mathrm{~s}$ and finally returning to display a neutral face for $1 \mathrm{~s}$. Each video clip lasted $5 \mathrm{~s}$ in total. In addition, pixelated videos were created (Photoshop CS3 v.10.0 ${ }^{\circledR}$ and Adobe Premiere Pro $\mathrm{CS}^{\circledR}{ }^{\circledR}$ ). In these pixelated videos, the actors' faces were scrambled into randomly moving squares, whereas the background remained original. A fixation cross either highlighted in red, blue, or green was presented on the scramble for the mid $3 \mathrm{~s}$ of the video to cue participants for execution of one of the three facial expressions (see below). The stimuli were presented with MR-compatible goggles (Resonance Technology, Inc., Northridge, CA, USA) using the Presentation (C) software package v.11.0 (Neurobehavioral Systems, Inc., Albany, CA, USA).

The $\mathrm{fMRI}$ experiment was planned as a $3 \times 2$ factorial design with factors "facial expression" (emotional, non-emotional, neutral face) and "task" (execution, observation). Participants were instructed to (i) attentively observe the videos displaying actors or (ii) in case of pixelated video clips to execute a facial expression. The kind of facial expression (emotional/smile, non-emotional/ lip protrusion, neutral/relaxed face) was designated by the color of the fixation cross. The participants were to execute the facial expression as long as the fixation cross showed up. Participants' facial expressions were filmed with a scanner-compatible camera and monitored online. The measurement was interjected in case of repeated errors (e.g., participant executed a wrong facial expression or mimicked the actor's face during observation). The participant was then reinstructed and the run was restarted (this happened in case of two patients and one healthy control).

Immediately after the fMRI measurement, participants were asked to rate their subjective feeling of happiness during each condition (PSQ). 


\section{Demographic and Behavioral Data Analyses}

We analyzed behavioral data using IBM SPSS Statistics version 21. The proportional FEEST data were arcsine root square transformed before comparison of mean values by calculating a repeated measures ANOVA (50). Normal distribution of demographic and PSQ data was checked by evaluating skewness and kurtosis of these data in the two groups (51). A variable was assumed normally distributed when skewness [S = skewness/SE (skewness)] and kurtosis $[K=$ kurtosis/SE (kurtosis)] were not greater than \pm 2 . Because normality was assumed for the PSQ and most demographic variables, repeated measures ANOVAs and/or (post hoc) independent $t$-tests $(p<0.05$, two-tailed) were calculated (see also Section "Results" and the Section "Procedure and Participants"). The assumption of normal distribution was violated concerning the age of participants. Therefore, a Mann-Whitney $U$-test was calculated to compare the age of the two groups (52). Post hoc pairwise comparisons were Bonferroni-corrected $(p=0.05 /$ number of tests).

\section{fMRI Data Acquisition and Analyses}

All fMRI data analyses were performed with SPM8 (Wellcome Department of Imaging Neuroscience, London, UK) implemented in Matlab 8.1 (Mathworks Inc., Sherborn, MA, USA).

\section{fMRI Data Acquisition, Preprocessing, and Single Subject Analyses}

We obtained anatomical and functional images with a Siemens 3T Trio MR-scanner. Functional T2* weighted echo-planar images (EPIs) were obtained with the following parameters: $\mathrm{TR}=2,400 \mathrm{~ms}, \mathrm{TE}=30 \mathrm{~ms}$, flip angle $=90^{\circ}, \mathrm{FoV}=240 \mathrm{~mm}$, matrix size $=64 \times 64$, in-plane resolution $=3.8 \mathrm{~mm} \times 3.8 \mathrm{~mm}$, 36 slices with slice thickness $3.0 \mathrm{~mm}$, and distance factor $3 \%$. A high-resolution T1-weighted anatomical 3-D magnetization prepared rapid gradient echo image $(\mathrm{TR}=1,900 \mathrm{~ms}, \mathrm{TE}=2.52 \mathrm{~ms}$, $\mathrm{TI}=900 \mathrm{~ms}$, flip angle $=9^{\circ}, \mathrm{FoV}=250 \mathrm{~mm}, 256 \times 256$ matrix, 176 slices per slab) was recorded.

The first five EPI volumes were discarded to allow for T1 equilibration effects. The remaining functional images were realigned to the first image to correct for head motion (53). Participants, except for one, moved less than $4.1 \mathrm{~mm}$ (translation) and $4.6^{\circ}$ (rotation). One patient had a rotation value of $14^{\circ}$. Therefore, movement parameters were included as six additional regressors into the general linear model (GLM) as covariates of no interest to model variance related to absolute head motion. Prior to that, for each participant, the T1 image was co-registered to the mean image of the realigned functional images. The mean functional image was normalized to the MNI template [Montreal Neurological Institute $(54,55)]$, using a segmentation algorithm (56). Normalization parameters were applied to all EPI images and the T1 image. The images were resampled to $1.5 \mathrm{~mm} \times 1.5 \mathrm{~mm} \times 1.5 \mathrm{~mm}$ voxel size and spatially smoothed with an $8 \mathrm{~mm}$ full width half maximum isotropic Gaussian kernel.

Data were subsequently analyzed by a two-level approach. Using a GLM, each experimental condition (emotional observation, non-emotional observation, neutral observation, emotional execution, non-emotional execution, neutral execution) was modeled on the single-subject level with a separate regressor convolved with a canonical hemodynamic response function and its first temporal derivative. The parameter estimates for each voxel were calculated using maximum likelihood estimation and corrected for non-sphericity.

We calculated two second-level models, one to replicate previous findings on shared representations of facial expressions and one to identify brain activation differences of PD and HC. As a proof of concept, we identified shared representations for the emotional and the non-emotional facial expressions the current sample first. Following the analyses of our previous study in healthy participants (34), the neutral/static facial expression was conceptualized as high-level baseline. Activation during observation and execution of neutral facial expressions was subtracted on the single subject level: Observation: emotional minus neutral facial expression (E_OBS minus N_OBS) and non-emotional minus neutral facial expression (NE_OBS minus N_OBS); execution: emotional minus neutral facial expression (E_EXE minus N_EXE) and non-emotional minus neutral facial expression (NE_EXE minus N_EXE). Resulting four contrast images for each group (HC, PD) were fed into a flexible factorial second-level analysis using a one-way four-level ANOVA (factor: condition; blocking factor: subject). To identify shared representations for execution and observation, we calculated four conjoint conjunction analyses across the corresponding execution and observation contrast, separately for HC and PD and for the emotional and the non-emotional facial expression. In our previous study examining shared representations for happy and non-emotional facial expressions in healthy participants, we found significant differences between happy and nonemotional facial gestures, which we attributed to the enhanced communicative information of the emotional facial expression. We expected to replicate this previous finding here.

The second model was computed to assess differences between $\mathrm{HC}$ and PD. Here, we were especially interested in the communicative emotional facial gesture. All six first-level contrasts for each group (E_OBS, NE_OBS, N_OBS, E_EXE, NE_EXE, N_EXE) were fed into a flexible factorial analysis using a one-way 12-level ANOVA (factor: condition; blocking factor: subject). Four contrasts were computed: E_EXE: HC minus PD, HC minus PD; E_OBS: HC minus, HC minus PD. All contrasts were masked inclusive with OBS and EXE of the $\mathrm{HC}(t>3.14)$ to restrict the analysis to brain regions activated during execution and observation, respectively, of emotional facial expressions in HC. Group comparisons were restricted to the emotional facial expression, but effects of interest plots were generated to display activation of areas of interest during all conditions. We obtained a mask, encompassing the right IFGop from the AAL atlas embedded in the WFU PickAtlas [Wake Forest University, WinsotnSalem, NC, USA (57)] as IFGop was the region of most interest derived from the literature. Results of regions of interest analyses are reported FWE-corrected at a threshold of $p<0.05$. Further exploratory whole brain analyses are reported at a threshold of $p<0.001$, uncorrected, with an extent threshold of $k>10$ voxel, to consider the small sample size. Brain structures were labeled using the Anatomy Toolbox v $1.6(58,59)$. 
We extracted brain data from all clusters in the IFGop or IPL that were identified in the group comparison, because these two regions were the homologs of monkey mirror areas. We selected the eigenvariate option (adjusted for the effects of interest). Pearson's correlation coefficient was used to assess the relation of brain data and emotion recognition in the FEEST as well as medication [LEDD (40)] and disease duration in PD, with results being reported significant at a threshold of $p<0.05$ (two-tailed).

\section{RESULTS}

\section{Behavioral Data}

Means and SDs of the PSQ and the emotion recognition accuracies in the FEEST are presented in Table 1.

A $2 \times 2 \times 6$ repeated measures ANOVA with within-subject factors "difficulty" (90-70 and 70-30\%) and "emotion" (happiness, surprise, fear, sadness, disgust, anger) and between-subject factor "group" revealed a significant main effect of difficulty $\left(F_{1,24}=20.30, p<0.001\right)$ and a significant main effect of emotion $\left(F_{5,120}=12.45, \varepsilon=0.57, p<0.001\right)$. There was neither a significant main effect of group $\left(F_{1,24}=1.10, p=0.306\right)$, nor any significant interaction effect (all $p \geq 0.328$ ). A separate analysis was run for the $50-50 \%$ morphs because responses to these morphs were counted as correct if the participant recognized either of the two emotions contained in the morph. For the 50-50\% morphs, PD performed significantly worse than $\mathrm{HC}\left(t_{24}=2.43, p=0.023\right)$.

Post-scanning ratings were analyzed with a repeated measures ANOVA with "facial expression" (emotional, non-emotional, neutral) and "task" (observe, execute) as within-subject factors

TABLE 1 | Post-scanning questionnaire (PSQ) and emotion recognition test [Facial Expressions of Emotions-Stimuli and Test battery (FEEST)].

\begin{tabular}{|c|c|c|c|c|}
\hline \multirow[b]{2}{*}{ PSQ } & \multicolumn{2}{|c|}{ HC } & \multicolumn{2}{|c|}{ PD patients } \\
\hline & $N$ & M (SD) & $N$ & M (SD) \\
\hline E_OBS & 13 & $3.77(1.69)$ & 13 & $3.46(1.71)$ \\
\hline NE_OBS & 13 & $2.92(1.32)$ & 13 & $3.00(2.08)$ \\
\hline N_OBS & 13 & $2.23(1.24)$ & 12 & $2.67(1.23)$ \\
\hline E_EXE & 13 & $4.15(1.07)$ & 13 & $4.15(2.12)$ \\
\hline NE_EXE & 13 & $2.92(1.55)$ & 13 & $3.92(2.06)$ \\
\hline N_EXE & 13 & $2.77(1.64)$ & 13 & $2.62(1.89)$ \\
\hline \multirow[t]{2}{*}{ FEEST } & \multicolumn{2}{|c|}{$\begin{array}{c}\text { HC M (SD) } \\
\quad N=13\end{array}$} & \multicolumn{2}{|c|}{$\begin{array}{l}\text { PD patients M (SD), } \\
\qquad N=13\end{array}$} \\
\hline & $90-10 \%$ & $70-30 \%$ & $90-10 \%$ & $70-30 \%$ \\
\hline Happiness & $9.85(0.38)$ & $9.77(0.6)$ & $9.85(0.38)$ & $9.15(1.14)$ \\
\hline Surprise & $8.15(2.64)$ & $7.77(1.64)$ & $8.54(1.95)$ & $7.77(1.64)$ \\
\hline Fear & $8.0(2.42)$ & $7.31(1.7)$ & $7.00(2.27)$ & $5.23(2.77)$ \\
\hline Sadness & $9.77(0.6)$ & $9.54(0.66)$ & $8.92(1.38)$ & $8.08(2.53)$ \\
\hline Disgust & $7.13(4.12)$ & $6.85(3.93)$ & $7.38(3.25)$ & 6.69 (3.09) \\
\hline Anger & $8.46(2.37)$ & $7.85(2.38)$ & $8.77(1.69)$ & $7.92(2.63)$ \\
\hline $50-50 \%$ & \multicolumn{2}{|c|}{$26.77(3.0)$} & \multicolumn{2}{|c|}{$24.08(3.35)$} \\
\hline
\end{tabular}

Means $(M)$ and $S D$ for the post-scanning questionnaire (top) and emotion recognition test (bottom).

E_OBS, emotional observation; NE_OBS, non-emotional observation; N_OBS, neutral observation; E_EXE, emotional execution; NE_EXE, non-emotional execution; N_EXE, neutral execution; HC, healthy controls; PD, Parkinson's disease; mean hits are displayed for the FEEST (90-10 and 70-30\% condition consisted of 10 trials the; 50-50\% condition consisted of 30 trials). and "group" as a between-subject factor. There was a significant main effect of facial expression $\left(F_{2,46}=12.79, p<0.001\right)$ and a trend for the main effect task $\left(F_{1,23}=3.42, p=0.077\right)$, but no significant main effect of group $(p=0.637)$ and no significant two or three-way interaction effect (all $p>0.32$ ). Three post hoc tests were calculated resulting in a Bonferroni-corrected threshold $p=0.017$. Participants rated their happiness higher after production and observation (values of both tasks were averaged) of emotional compared to non-emotional $\left(t_{25}=2.86\right.$, $p=0.008)$ or neutral facial expressions $\left(t_{25}=5.77, p<0.001\right)$. The difference of happiness ratings between the non-emotional and neutral facial expression did not survive the Bonferroni corrected threshold of $p=0.017\left(t_{25}=2.38, p=0.025\right)$.

\section{fMRI Data}

In the fMRI results section, brain regions are labeled according to Anatomy Toolbox $(58,59)$ with cytoarchitectonic areas listed in brackets.

\section{Manipulation Check: Shared Representations during Execution and Observation of Facial Expressions}

For both groups, conjoint conjunctions for execution and observation of the emotional facial expressions was found in bilateral precentral gyrus extending to right IFGop, right IPL (PF), right STG, and bilateral posterior middle temporal gyrus (MTG). HC additionally activated the bilateral (pre-)SMA, bilateral middle cingulate cortex, left supramarginal gyrus, right inferior temporal gyrus, right area PFt of the IPL and left cerebellum [VI (Hem), VIIa Crus I (Hem)] (see Figure 1). For patients, right frontal activation extended into the pars triangularis of the IFG and temporo-parietal activation extended into V5. Furthermore, right fusiform gyrus was activated in patients.

Conjoint activation during execution and observation of the non-emotional facial expression was found in the bilateral MTG in the conjunction analysis for each group. In HC, bilateral temporal cortex activation included also bilateral STG and right ITG. Furthermore, right IPL [intraparietal sulcus (IPS) hIP2, bilateral hIP3, supramarginal gyrus PF, PFm, PFcm, PFop], left posterior IPL, bilateral superior parietal lobule (SPL, 7PC), bilateral precentral gyrus (BA6), and bilateral cerebellum [VIIa Crus I (Hem), right VI (Hem)] were involved in HC. In PD patients, the conjunction revealed activation of the bilateral middle frontal gyrus (MFG), the bilateral posterior MTG activation extended area V5 (hOc5) and the left precentral gyrus (Table 2).

\section{Group Comparisons}

The region of interest analyses revealed decreased activation of the right IFGop during observation of the emotional facial expression in PD compared to HC [MNI (54 14 37), $t=4.99$, $k=3, p<0.001$ FWE-corrected and MNI (54 17 19), $t=3.56$, $k=3, p<0.001$ FWE-corrected]. Consequentially, decreased right IFGop activation was found in the exploratory whole brain analysis, too, but in this analysis extended to the right precentral gyrus. The exploratory whole brain analysis furthermore revealed stronger activation of right IPL (BA 1, BA2, BA40, PFt of the supramarginal gyrus, hIP3 of the IPS) and left IPL (BA2, PFt), bilateral (pre-) SMA, and bilateral thalamus in HC compared to 


\section{Emotional}

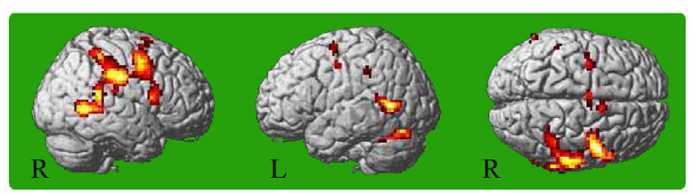

Non-Emotional

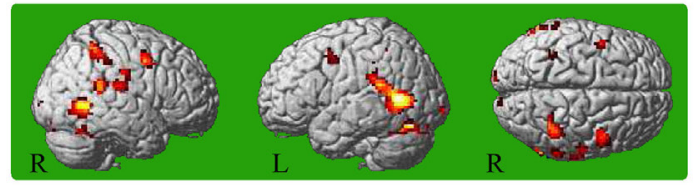

\section{Emotional}

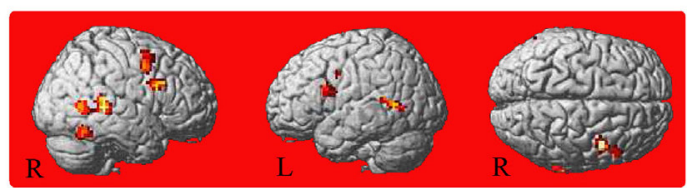

Non-Emotional

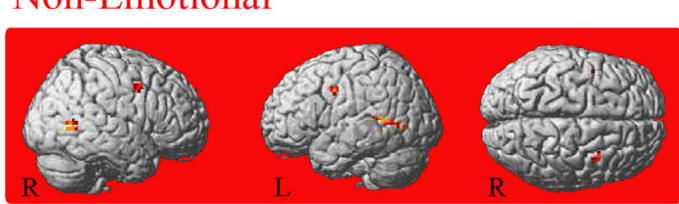

FIGURE 1 | Conjoint activation for the observation and execution of facial expressions. Shared representations for observation and execution of facial expressions. Data of healthy controls are highlighted in green, data of patients with Parkinson's disease in red. Abbreviations: HC, healthy controls; PD, patients with Parkinson's disease; E_OBS, observation of emotional facial expressions; E_EXE, execution of emotional facial expressions; NE_OBS, observation of non-emotional facial expressions; NE_EXE, execution of non-emotional facial expressions. The conjunction is thresholded at $p<0.001$ (uncorrected, $k \geq 10$ voxel).

PD during observation of the emotional facial expression (see Figure 2 and for a comprehensive enumeration, see Table 2).

Patients with PD did not show stronger activity than HC in any brain region in this condition.

Execution of emotional facial expressions resulted in increased activation of the right putamen in patients with $\mathrm{PD}$ compared to $\mathrm{HC}$.

\section{Correlation of Brain Activity, Emotion Recognition Accuracies, and LEDD Scores}

In line with our hypotheses, we identified group differences in the IFG and IPL during observation of the emotional facial expression. We correlated brain activation in these regions during observation of the emotional facial expressions (E_OBS-N_OBS) with the emotion recognition accuracies for PD and HC separately. We used mean accuracy across difficulties (90-10, 70-30\%) and across emotions as well as accuracy data concerning the 50-50\% morphs. Finally, brain activation (E_OBS-N_OBS) was correlated with LEDD. In $\mathrm{PD}$, we found a significant positive relation of right IPL activity and emotion recognition accuracy $[r(11)=0.61$, $p=0.026]$ and a trend for a positive correlation of right IPL activity and emotion recognition concerning the ambiguous he $50-50 \%$ morphs $(r(11)=0.51, p=0.077)$ in PD patients. No significant correlations were observed in HC (correlation of right IPL activity and mean emotion recognition: $[r(11)=0.2$, $p=0.504]$; and of right IPL activity and emotion recognition concerning the $50-50 \%$ morphs: $[r(11)=0.15, p=0.62])$. We found no significant correlation of right IFGop activity with mean accuracy [PD: $r(11)=0.4, p=0.17$; HC: $r(11)=0.25$, $p=0.41]$ or with accuracy concerning the $50-50 \%$ morphs [PD: $r(11)=0.28, p=0.35$; HC: $r(11)=0.17, p=0.59$ ]. The same was true for the correlation of left IPL and mean accuracy [PD: $r(11)=0.4, p=0.17$; HC: $r(11)=0.3, p=0.31$ ] as well as accuracy concerning the $50-50 \%$ morphs [PD: $r(11)=0.2$, $p=0.5$; HC: $r(11)=0.14, p=0.64]$. There was no relation of medication and IFGop or IPL activity.

\section{DISCUSSION}

We examined emotion recognition abilities and underlying brain mechanisms in PD and HC. Both groups performed similarly well in the emotion recognition task, when ambiguousness of morphs was low. Because accuracy rates were high for 90/10 and $70 / 30 \%$ morphs, these morphs might have been too easy to recognize, to reveal group differences. In line with this assumption, patients had slightly more difficulties to recognize highly ambiguous facial expressions (50\% morphs of two similar emotions). A meta-analysis on emotion recognition in PD revealed an impairment with a clinical significant medium but heterogeneous effect size in patients, with task difficulty assumed as possible influence factor (46). In line with this, FEEST tests had not the power to reveal deficits in a small sample of PD in a previous study (60), but differences were detected with a refined assessment of emotion recognition. As expected, both $\mathrm{PD}$ and $\mathrm{HC}$ activated inferior frontal and anterior inferior parietal human homologs of mirror neuron areas during execution and observation of emotional facial expression. As predicted, we found a significantly decreased response of fronto-parietal human homologs of mirror neuron areas in patients.

\section{IFGop}

Both groups activated the right IFGop during execution and observation of emotional faces, but the response of the dorsal section of the IFGop and adjacent rostral part of the dorsal premotor cortex was reduced during observation of the emotional facial expression in PD relative to HC. Supposed to be the human homolog of monkey premotor mirror neuron area F5 (23), the IFGop has consistently been shown to be activated during observation and imitation of goal-directed movements (61) and processing of facial expressions (62). In our previous studies in healthy participants (34) and in Parkin mutation carriers (10), using a similar experimental design (but another scanning protocol and sample) conjoint activation for execution and observation of emotional facial expression was found inferior to the weaker than normal IFGop activation 
TABLE 2 | Brain activation clusters for all whole brain contrasts.

\begin{tabular}{|c|c|c|c|c|c|c|c|c|c|}
\hline \multirow[b]{2}{*}{ Contrast } & & \multicolumn{4}{|c|}{ MNI } & \multirow[b]{2}{*}{$t$-Value } & \multirow[b]{2}{*}{$p$-Value } & \multirow[b]{2}{*}{ Side } & \multirow[b]{2}{*}{ Region } \\
\hline & & $x$ & $Y$ & $z$ & $k$ & & & & \\
\hline \multirow[t]{9}{*}{ E_OBS $\cap$ E_EXE } & $\mathrm{HC}$ & 48 & 2 & 46 & 1,050 & 6.14 & $<0.001$ & $r$ & Precentral gyrus \\
\hline & & -27 & -73 & -23 & 157 & 5.33 & $<0.001$ & 1 & Cerebellum \\
\hline & & -48 & -58 & 4 & 166 & 5.16 & $<0.001$ & 1 & Middle temporal gyrus (MTG) \\
\hline & & -33 & -4 & 64 & 15 & 4.41 & $<0.001$ & 1 & Precentral gyrus \\
\hline & & -45 & -37 & 34 & 13 & 4.16 & $<0.001$ & 1 & Supramarginal gyrus \\
\hline & & -39 & -7 & 46 & 19 & 3.88 & $<0.001$ & I & Precentral gyrus \\
\hline & & 9 & 8 & 67 & 25 & 3.80 & $<0.001$ & r & Pre-supplementary motor area \\
\hline & & -6 & -7 & 64 & 26 & 3.78 & $<0.001$ & $b$ & Supplementary motor area \\
\hline & & -3 & 11 & 43 & 30 & 3.65 & $<0.001$ & $\mathrm{~b}$ & Midcingulate cortex \\
\hline \multirow[t]{7}{*}{ E_OBS $\cap$ E_EXE } & PD & 45 & -52 & -20 & 66 & 4.92 & $<0.001$ & r & Fusiform gyrus \\
\hline & & 45 & 11 & 25 & 85 & 4.55 & $<0.001$ & $r$ & Inferior frontal gyrus pars opercularis \\
\hline & & 42 & 5 & 43 & 59 & 4.55 & $<0.001$ & r & Precentral gyrus \\
\hline & & 51 & -61 & 7 & 157 & 4.31 & $<0.001$ & $r$ & MTG \\
\hline & & -42 & 8 & 19 & 45 & 3.96 & $<0.001$ & 1 & Inferior frontal gyrus pars opercularis \\
\hline & & -54 & -55 & 7 & 58 & 3.95 & $<0.001$ & 1 & MTG \\
\hline & & -39 & 2 & 37 & 11 & 3.74 & $<0.001$ & 1 & Precentral gyrus \\
\hline \multirow[t]{15}{*}{ NE_OBS $\cap$ NE_EXE } & $\mathrm{HC}$ & -48 & -58 & 4 & 572 & 6.89 & $<0.001$ & I & MTG \\
\hline & & 36 & -46 & 52 & 154 & 5.12 & $<0.001$ & r & Inferior parietal lobule (hiP3) \\
\hline & & 51 & -61 & 1 & 183 & 5.06 & $<0.001$ & r & MTG \\
\hline & & 57 & -40 & 19 & 75 & 4.90 & $<0.001$ & r & Superior temporal gyrus \\
\hline & & -27 & -70 & -20 & 260 & 4.77 & $<0.001$ & 1 & Cerebellum VI (Hem) \\
\hline & & 63 & -22 & 19 & 67 & 4.60 & $<0.001$ & r & Supramarginal gyrus \\
\hline & & 42 & -55 & -26 & 36 & 4.52 & $<0.001$ & r & Cerebellum VI (Hem) \\
\hline & & 45 & 2 & 43 & 67 & 4.21 & $<0.001$ & r & Precentral gyrus (BA 6) \\
\hline & & -45 & 2 & 46 & 25 & 4.16 & $<0.001$ & 1 & Precentral gyrus (BA 6) \\
\hline & & 21 & -85 & -20 & 20 & 3.98 & $<0.001$ & 1 & Cerebellum VIla (Hem) \\
\hline & & -12 & -103 & -5 & 15 & 3.71 & $<0.001$ & 1 & Calcarine gyrus \\
\hline & & -27 & 5 & -23 & 15 & 3.66 & $<0.001$ & I & Temporal pole \\
\hline & & -30 & -46 & 52 & 10 & 3.48 & $<0.001$ & 1 & Inferior parietal lobule (BA 2) \\
\hline & & 15 & -97 & 4 & 10 & 3.46 & $<0.001$ & r & Calcarine gyrus \\
\hline & & -9 & -16 & 1 & 10 & 3.44 & $<0.001$ & 1 & Thalamus (prefrontal) \\
\hline \multirow[t]{4}{*}{ NE_OBS $\cap$ NE_EXE } & PD & 36 & 8 & 40 & 16 & 4.04 & $<0.001$ & r & Middle frontal gyrus (MFG) \\
\hline & & 51 & -64 & 4 & 16 & 3.94 & $<0.001$ & $r$ & Middle temporal gyurs \\
\hline & & -45 & -1 & 40 & 13 & 3.86 & $<0.001$ & 1 & Precentral gyrus \\
\hline & & -54 & -52 & 7 & 34 & 3.80 & $<0.001$ & 1 & MTG \\
\hline \multirow[t]{12}{*}{ E_OBS ${ }^{a}$} & $\mathrm{HC}>\mathrm{PD}$ & 12 & -97 & 4 & 3,480 & 8.73 & $<0.001$ & $b$ & Calcarine gyrus \\
\hline & & 36 & -49 & 49 & 515 & 7.48 & $<0.001$ & r & Inferior parietal lobule hiP3 \\
\hline & & 54 & 14 & 37 & 42 & 4.99 & $<0.001$ & r & Inferior frontal gyrus \\
\hline & & 18 & -25 & -5 & 33 & 4.88 & $<0.001$ & r & Thalamus \\
\hline & & 33 & 2 & 64 & 15 & 4.47 & $<0.001$ & r & Superior frontal gyrus \\
\hline & & -48 & -25 & 40 & 11 & 4.13 & $<0.001$ & 1 & Inferior parietal lobule \\
\hline & & 36 & -7 & 40 & 24 & 4.00 & $<0.001$ & r & Precentral gyrus \\
\hline & & -21 & -7 & 1 & 17 & 3.99 & $<0.001$ & I & Pallidum \\
\hline & & 0 & 11 & 58 & 31 & 3.80 & $<0.001$ & $\mathrm{~b}$ & Supplementary motor area \\
\hline & & 27 & -31 & 70 & 16 & 3.64 & $<0.001$ & r & Precentral gyrus \\
\hline & & 48 & 47 & 4 & 10 & 3.57 & $<0.001$ & I & MFG \\
\hline & & -24 & -22 & 4 & 18 & 3.54 & $<0.001$ & 1 & Thalamus (parietal, motor, somatosensory) \\
\hline E_OBS ${ }^{a}$ & $\mathrm{PD}>\mathrm{HC}$ & - & - & - & - & - & - & - & - \\
\hline E_EXE ${ }^{a}$ & $\mathrm{HC}>\mathrm{PD}$ & - & - & - & - & - & - & - & - \\
\hline E_EXE & $\mathrm{PD}>\mathrm{HC}$ & 24 & -1 & 7 & 21 & 4.06 & $<0.001$ & & Putamen \\
\hline
\end{tabular}

MNI coordinates of the main peaks of significant clusters at a threshold of $p<0.001$ uncorrected, the number of significant voxel ( $k$ ), Table includes furthermore the hemisphere ( $L=$ left, $R=$ right, and $B=$ bilateral) of the cluster, and the name of the region in which the main peak was localized.

$H C$, healthy controls; $P D$, patients suffering Parkinson's disease; E, emotional; NE, non-emotional; OBS, observation; EX, execution.

${ }^{a}$ All group comparison contrasts were masked inclusive with E_OBS and E_EXE of HC $(p<0.05)$.

in PD in the current study. However, other studies found dorsal IFGop activated during observation $(33,63,64)$ as well as during execution of emotional facial expressions (33) remarkably close to the peak in the present study. Activation of dorsal IFGop during observation of facial expressions was thought to represent activation of the mouth motor area (32) due to preparation of an automatic motor response, "facial mimicry" or a resonant activation of a dorsal sector of the mirror neuron system $(33,65,66)$. Thus, 

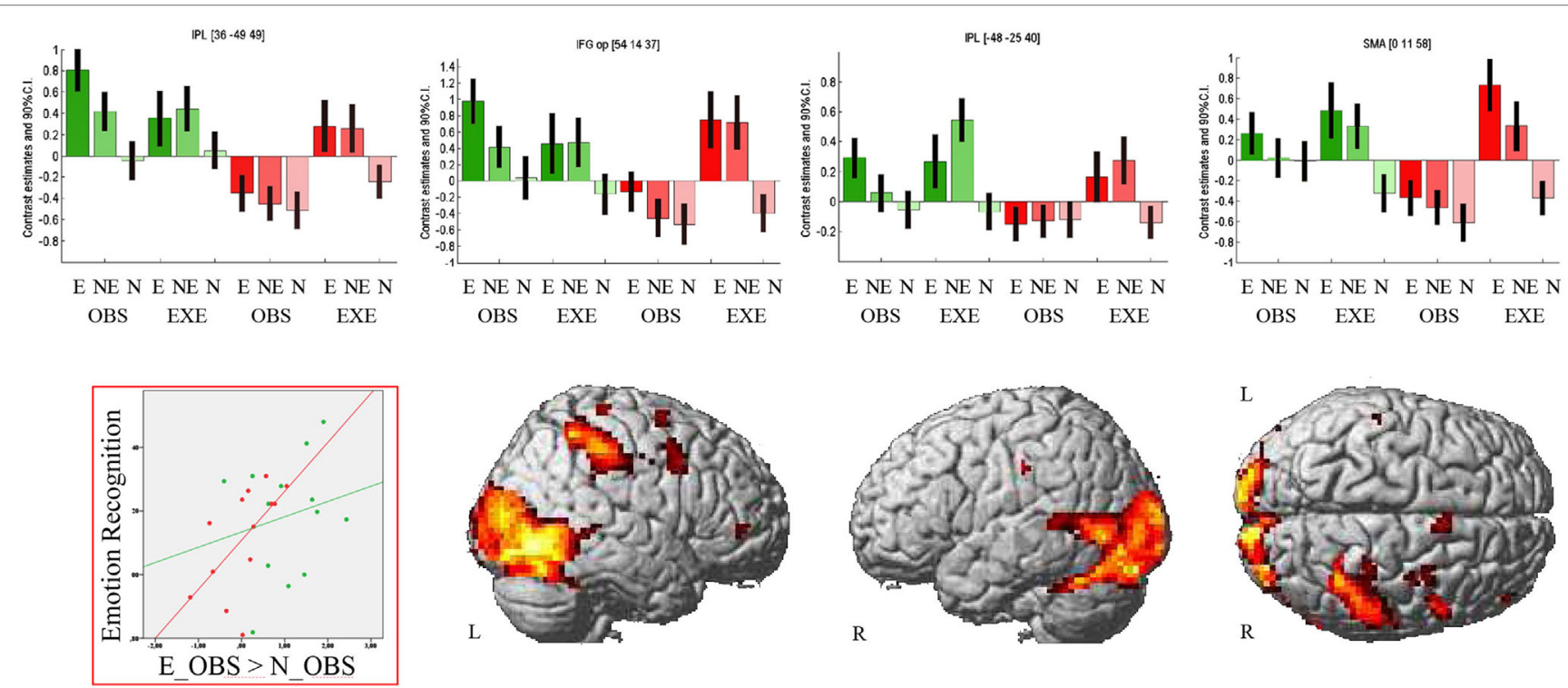

FIGURE 2 | Observation of emotional facial expressions: Group comparison. Activation during observation of the emotional facial expression in healthy controls contrasted with activation during the same condition in patients with Parkinson's disease. Bar graphs show brain activation level of human 'mirror' areas for all conditions: E, emotional; NE, non-emotional; N, neutral; OBS, observation; EXE, execution; healthy controls are colored in green, patients with Parkinson's disease in red. The scatterplot depicts the relation of emotion recognition accuracy (Mean of $90-10 \%$ and $70-30 \% \mathrm{morphs}, \mathrm{rPD}(11)=0.61, p=0.026$; $r \mathrm{HC}(11)=0.2$, $p=0.504$ ) and activation of the right inferior parietal lobule (contrast E_OBS-N_OBS). The contrast is thresholded at $p<0.001$ (uncorrected, $k \geq 10$ voxel).

reduced muscular mimicry accompanied by reduced resonance in the IFGop might account for emotion recognition deficits in PD. Besides this, lowered right dorsal IFGop activation was related with self-reported difficulties in identifying one's own feelings (Alexithymia) in a recent study (67), and PD have been shown to suffer from Alexithymia double as often as control subjects (68). Thus, this region might also be important for the understanding of one's own feelings. Interestingly, in contrast to Parkin mutation carriers (10), emotion recognition accuracy in PD with manifest Parkinson's disease was not related to IFGop activity in the current study. Due to the small sample size, we had not enough power to detect small to medium size relations. Moreover, the FEEST might have been too easy to uncover emotion recognition deficits in PD. Future studies might use a more difficult emotion recognition test to enhance variance of emotion recognition accuracy and increase the sensitivity to detect relations between IFGop activation and behavioral performance.

\section{Parietal Cortex}

We found widespread weaker than normal activation in patients with PD in the right IPL including supramarginal gyrus (PFt), somatosensory cortices, and the anterior IPS and a more localized decrease of activation in the left supramarginal gyrus (PFt) and somatosensory cortex.

Decreased activation of the supramarginal area PFt is in line with our hypothesis of a disturbed resonance in homologs of monkey mirror areas in $\mathrm{PD}$, in contrast to stronger involvement of supramarginal gyri as shared representation of execution and observation of facial affect in HC [concerning HC, see also (69)]. Furthermore, somatosensory cortices [bilateral SI and SII (22), left SII (32), right SII (33)] have previously been shown to be activated during observation and execution/imitation of emotional facial expressions (but see two studies that did not find overlapping activity in the inferior parietal lobules 31,34 ). In accordance with the positive relation of right IPL activation and emotion recognition accuracy in patients with $\mathrm{PD}$ in the current study, lesions of right IPL encompassing somatosensory areas and supramarginal gyrus have previously been reported to cause emotion recognition deficits (70). Furthermore, abnormal activation and connectivity patterns of the right IPL in patients with PD were shown in previous resting state studies $(71,72)$. Decreased connectivity of IPL and primary motor cortex and SMA was supposed to reflect a disturbance in networks linked to motor preparation and initiation in PD (73).

The IPS was assumed to be part of a frontoparietal network involved in adaptive online control of actions (74-76) and coding of action goals (75). Self- and other-generated actions are commonly represented in the IPS (75). Right IPS involvement was reported to be selective for face compared to object processing (62), shown during short presentation of facial expressions (77) and during emotion differentiation (78).

\section{Supplementary Motor Area}

Analogously to our antecedent study (34), HC activated the right (pre-)SMA during observation and execution of emotional facial expressions. Interestingly, activation of this region was significantly decreased in PD patients. The Pre-SMA is involved in motor preparation (79). Activation during observation might, therefore, represent a starting contagious motor response of the observers face. Deviant activation in PD might result from functional changes in the basal ganglia-cortical motor loops, as pre-SMA is interconnected with the striatum (80) and the 
subthalamic nucleus (81), which in turn is connected to globus pallidus. The weaker activity in patients with $\mathrm{PD}$ compared to $\mathrm{HC}$ in our study is in accordance with a previously reported decreased brain activation (indexed through the amplitude of low frequency fluctuations) in the SMA during resting state (82) and an increase of activation during mental simulation of actions when PD patients were on compared to off dopaminergic medication (1).

\section{Limitations}

We examined emotion recognition and processing of emotional facial expressions in a small sample of medicated patients with PD. Given the small sample size, the power of our study is appropriate to detect large correlations, but error probability $\beta$ is high concerning medium and small effects (83). Furthermore, we measured medicated patients. And although our data are in accordance with and complement our findings in Parkin mutation carriers (10), and emotion recognition deficits in PD have been shown to be unrelated to dopaminergic medication (46), the results of this study have to be interpreted with caution until replication in larger samples and with unmedicated patients. Moreover, although similar brain activation patterns were shown during observation and execution of facial affect irrespective of which facial emotions were included $(22,32,33)$, we cannot generalize our brain activation results to other emotions. Future studies could test the relation of emotion recognition and brain activation during observation of diverse facial emotions.

\section{CONCLUSION}

We provide evidence for altered brain activation in manifest PD in human homologs of mirror neuron areas, which is partly linked to emotion recognition accuracy in PD. Visual input to the human mirror neuron system is supposed to be forwarded from posterior superior temporal sulcus to the parietal human homolog of monkey area PF/PFG where a motoric description is stored. Then, information is forwarded to IFGop and ventral premotor cortex, where action goals are coded (84). During imitation of actions, efference copies are sent back from frontal areas to IPL and from there to the posterior superior temporal sulcus. These efference copies allow a matching of sensory predictions of motor plans with the observed action (84). The transfer of information in this neural imitation circuitry might also be applicable to automatic facial mimicry. A disruption of information flow due to deactivation of frontal and parietal human homologs of mirror

\section{REFERENCES}

1. Peron J, Dondaine T, Le Jeune F, Grandjean D, Verin M. Emotional processing in Parkinson's disease: a systematic review. Mov Disord (2012) 27(2):186-99. doi: $10.1002 / \mathrm{mds} .24025$

2. Pentland B, Pitcairn TK, Gray JM, Riddle WJ. The effects of reduced expression in Parkinson's disease on impression formation by health professionals. Clin Rehabil (1987) 1(4):307-13. doi:10.1177/026921558700100410

3. Visser M, Verbaan D, van Rooden S, Marinus J, van Hilten J, Stiggelbout A. A longitudinal evaluation of health-related quality of life of patients with Parkinson's disease. Value Health (2009) 12(2):392-6. doi:10.1111/j.1524-4733. 2008.00430.x

4. DeLong MR, Wichmann T. Circuits and circuit disorders of the basal ganglia. Arch Neurol (2007) 64(1):20-4. doi:10.1001/archneur.64.1.20 neuron areas in PD may lead to a disruption of neural resonance and thus be the basis of impaired emotion recognition.

\section{ETHICS STATEMENT}

This study was carried out in accordance with the recommendations of the Declaration of Helsinki with written informed consent from all subjects. The study protocol was approved by the local Ethics Committee (Medical Faculty of the RWTH Aachen University; code: EK 099/08).

\section{AUTHOR CONTRIBUTIONS}

Author roles: (1) research project. (A) Conception: SA and AP conceptualized the study. AP developed the stimulus material and executed the rating and selection of stimuli. $\mathrm{KM}, \mathrm{FB}$, and $\mathrm{AP}$ were involved in applying for the ethics vote. $\mathrm{AP}$ and $\mathrm{FB}$ implemented the experiment for measurements with the fMRI scanner in Aachen. (B) Organization: FB and AP organized the study. KR and $\mathrm{JH}$ recruited patients, obtained medical history, and performed clinical examination. (C) Execution: AP screened participants, introduced them to study protocol, performed neuropsychological testing and a part of the fMRI measurements. HC and HP performed fMRI measurements and the emotion recognition task. (2) Statistical Analysis. (A) Design: AP and FB designed the data analyses. (B) Execution: AP, HC, and HP executed data analyses. JH calculated levodopa equivalence dose scores. (C) Review and critique: SA, $\mathrm{KR}$, and KM reviewed statistics. (3) Manuscript preparation. (A) AP wrote the first draft. (B) All other authors reviewed the draft and approved the final version.

\section{ACKNOWLEDGMENTS}

The authors thank all participants for their enduring collaboration and interest in this research.

\section{FUNDING}

Financial disclosure/conflict of interest and funding sources for the study: KR was funded by the German Federal Ministry of Education and Research (BMBF 01GQ1402). JH was supported by the START-Program of the Faculty of Medicine at the RWTH Aachen University, Germany (23/12 to KR). AP and FB were funded by the German Federal Ministry of Education and Research (BMBF 01GW0752).

5. Alexander GE, Delong MR, Strick PL. Parallel organization of functionally segregated circuits linking basal ganglia and cortex. Annu Rev Neurosci (1986) 9:357-81. doi:10.1146/annurev.ne.09.030186.002041

6. Pan PL, Song W, Shang HF. Voxel-wise meta-analysis of gray matter abnormalities in idiopathic Parkinson's disease. Eu J Neurol (2012) 19(2):199-206. doi:10.1111/j.1468-1331.2011.03474.x

7. Ibarretxe-Bilbao N, Junque C, Tolosa E, Marti M-J, Valldeoriola F, Bargallo N, et al. Neuroanatomical correlates of impaired decision-making and facial emotion recognition in early Parkinson's disease. Eur JNeurosci (2009) 30(6):1162-71. doi:10.1111/j.1460-9568.2009.06892.x

8. Lotze M, Reimold M, Heymans U, Laihinen A, Patt M, Halsband U. Reduced ventrolateral fMRI response during observation of emotional gestures related to the degree of dopaminergic impairment in Parkinson disease. J Cogn Neurosci (2009) 21(7):1321-31. doi:10.1162/jocn.2009.21087 
9. Wabnegger A, Ille R, Schwingenschuh P, Katschnig-Winter P, Kögl-Wallner M, Wenzel K, et al. Facial emotion recognition in Parkinson's disease: an fMRI investigation. PLoS One (2015) 10(8):e0136110. doi:10.1371/journal. pone. 0136110

10. Anders S, Sack B, Pohl A, Münte T, Pramstaller P, Klein C, et al. Compensatory premotor activity during affective face processing in subclinical carriers of a single mutant Parkin allele. Brain (2012) 135(4):1128-40. doi:10.1093/ brain/aws 040

11. Braak H, Braak E, Yilmazer D, Devos RAI, Jansen ENH, Bohl J, et al. Amygdala pathology in Parkinsons-disease. Acta Neuropathol (1994) 88(6): 493-500. doi:10.1007/BF00296485

12. Harding AJ, Stimson E, Henderson JM, Halliday GM. Clinical correlates of selective pathology in the amygdala of patients with Parkinson's disease. Brain (2002) 125(11):2431-45. doi:10.1093/brain/awf251

13. Ouchi Y, Yoshikawa E, Okada H, Futatsubashi M, Sekine Y, Iyo M, et al. Alterations in binding site density of dopamine transporter in the striatum, orbitofrontal cortex, and amygdala in early Parkinson's disease: compartment analysis for beta-CFT binding with positron emission tomography. Ann Neurol (1999) 45(5):601-10. doi:10.1002/1531-8249(199905) 45:5<601::AID-ANA8>3.0.CO;2-0

14. Tessitore A, Hariri AR, Fera F, Smith WG, Chase TN, Hyde TM, et al. Dopamine modulates the response of the human amygdala: a study in Parkinson's disease. J Neurosci (2002) 22(20):9099-103.

15. Bologna M, Fabbrini G, Marsili L, Defazio G, Thompson PD, Berardelli A. Facial bradykinesia. J Neurol Neurosur Psychiatry (2013) 84(6):681-5. doi:10.1136/jnnp-2012-303993

16. Marneweck M, Palermo R, Hammond G. Discrimination and recognition of facial expressions of emotion and their links with voluntary control of facial musculature in Parkinson's disease. Neuropsychology (2014) 28(6):917-28. doi:10.1037/neu0000106

17. Jacobs DH, Shuren J, Bowers D, Heilman KM. Emotional facial imagery, perception, and expression in Parkinsons-disease. Neurology (1995) 45(9): 1696-702. doi:10.1212/WNL.45.9.1696

18. Ricciardi L, Visco-Comandini F, Erro R, Morgante F, Bologna M, Fasano A, et al. Facial emotion recognition and expression in Parkinson's disease: an emotional mirror mechanism? PLoS One (2017) 12(1):e0169110. doi:10.1371/journal.pone.0169110

19. Livingstone SR, Vezer E, McGarry LM, Lang AE, Russo FA. Deficits in the mimicry of facial expressions in Parkinson's disease. Front Psychol (2016) 7:780. doi:10.3389/fpsyg.2016.00780

20. Argaud S, Delplanque S, Houvenaghel JF, Auffret M, Duprez J, Verin M, et al. Does facial amimia impact the recognition of facial emotions? An EMG study in Parkinson's disease. PLoS One (2016) 11(7):e0160329. doi:10.1371/ journal.pone. 0160329

21. Bologna M, Berardelli I, Paparella G, Marsili L, Ricciardi L, Fabbrini G, et al. Altered kinematics of facial emotion expression and emotion recognition deficits are unrelated in Parkinson's disease. Front Neurol (2016) 7:230. doi:10.3389/fneur.2016.00230

22. van der Gaag C, Minderaa RB, Keysers C. Facial expressions: what the mirror neuron system can and cannot tell us. Soc Neurosci (2007) 2(3-4):179-222. doi:10.1080/17470910701376878

23. Rizzolatti G, Craighero L. The mirror-neuron system. Annu Rev Neurosci (2004) 27:169-92. doi:10.1146/annurev.neuro.27.070203.144230

24. di Pellegrino G, Fadiga L, Fogassi L, Gallese V, Rizzolatti G. Understanding motor events: a neurophysiological study. Exp Brain Res (1992) 91(1):176-80. doi:10.1007/BF00230027

25. Gallese V, Fadiga L, Fogassi L, Rizzolatti G. Action recognition in the premotor cortex. Brain (1996) 119(Pt 2):593-609. doi:10.1093/brain/119.2.593

26. Rozzi S, Ferrari PF, Bonini L, Rizzolatti G, Fogassi L. Functional organization of inferior parietal lobule convexity in the macaque monkey: electrophysiological characterization of motor, sensory and mirror responses and their correlation with cytoarchitectonic areas. Eur J Neurosci (2008) 28(8):1569-88. doi:10.1111/j.1460-9568.2008.06395.x

27. Petrides M, Cadoret GV, Mackey S. Orofacial somatomotor responses in the macaque monkey homologue of Broca's area. Nature (2005) 435(7046): 1235-8. doi:10.1038/nature03628

28. Passingham RE, Chung A, Goparaju B, Cowey A, Vaina LM. Using action understanding to understand the left inferior parietal cortex in the human brain. Brain Res (2014) 1582:64-76. doi:10.1016/j.brainres.2014.07.035
29. Rozzi S, Calzavara R, Belmalih A, Borra E, Gregoriou GG, Matelli M, et al. Cortical connections of the inferior parietal cortical convexity of the macaque monkey. Cereb Cortex (2006) 16(10):1389-417. doi:10.1093/cercor/ bhj076

30. Iacoboni M. Visuo-motor integration and control in the human posterior parietal cortex: evidence from TMS and fMRI. Neuropsychologia (2006) 44(13):2691-26999. doi:10.1016/j.neuropsychologia.2006.04.029

31. Carr L, Iacoboni M, Dubeau MC, Mazziotta JC, Lenzi GL. Neural mechanisms of empathy in humans: a relay from neural systems for imitation to limbic areas. Proc Natl Acad Sci U S A (2003) 100(9):5497-502. doi:10.1073/ pnas. 0935845100

32. Leslie KR, Johnson-Frey SH, Grafton ST. Functional imaging of face and hand imitation: towards a motor theory of empathy. Neuroimage (2004) 21(2):601-7. doi:10.1016/j.neuroimage.2003.09.038

33. Hennenlotter A, Schroeder U, Erhard P, Castrop F, Haslinger B, Stoecker D, et al. A common neural basis for receptive and expressive communication of pleasant facial affect. Neuroimage (2005) 26(2):581-91. doi:10.1016/j. neuroimage.2005.01.057

34. Kircher T, Pohl A, Krach S, Thimm M, Schulte-Ruther M, Anders S, et al. Affect-specific activation of shared networks for perception and execution of facial expressions. Soc Cogn Affect Neurosci (2012) 8(4):370-7. doi: $10.1093 /$ scan $/$ nss 008

35. Klein C, Lohmann-Hedrich K, Rogaeva E, Schlossmacher MG, Lang AE. Deciphering the role of heterozygous mutations in genes associated with parkinsonism. Lancet Neurol (2007) 6(7):652-62. doi:10.1016/S1474-4422 (07)70174-6

36. Guo JF, Wang L, He D, Yang QH, Duan ZX, Zhang XW, et al. Clinical features and [11C]-CFT PET analysis of PARK2, PARK6, PARK7-linked autosomal recessive early onset parkinsonism. Neurol Sci (2011) 32(1):35-40. doi:10.1007/s10072-010-0360-z

37. Buhmann C, Binkofski F, Klein C, Buchel C, van Eimeren T, Erdmann C, et al. Motor reorganization in asymptomatic carriers of a single mutant Parkin allele: a human model for presymptomatic parkinsonism. Brain (2005) 128:2281-90. doi:10.1093/brain/awh572

38. Demal U. SKIDPIT-Light Screeningbogen. Wien: Universität Wien (1999).

39. Oldfield RC. The assessment and analysis of handedness: the Edinburgh inventory. Neuropsychologia (1971) 9(1):97-113.

40. Tomlinson CL, Stowe R, Patel S, Rick C, Gray R, Clarke CE. Systematic review of levodopa dose equivalency reporting in Parkinson's disease. Mov Disord (2010) 25(15):2649-53. doi:10.1002/mds.23429

41. Folstein MF, Folstein SE, McHugh PR. Mini-mental state - practical method for grading cognitive state of patients for clinician. J Psychiatr Res (1975) 12(3):189-98. doi:10.1016/0022-3956(75)90026-6

42. Fahn S, Elton RL. Unified parkinsons disease rating scale. In: Fahn S, Marsden CD, Goldstein M, Calne DB, editors. Recent Developments in Parkinsons Disease. (Vol. 2), Florham Park, NJ: Macmillan Healthcare Information (1987). p. 153-63.

43. Lehrl S. Der MWT- ein Intelligenztest für die ärztliche Praxis. Praxis Neurologie und Psychiatrie (1996) 7:488-91.

44. Young AW, Perrett DI, Calder AJ, Sprengelmeyer R, Ekman P. Facial Expressions of Emotions: Stimuli and Test (FEEST). Thurstone: Thames Valley Test Company (2002).

45. Ekman P, Friesen WV. Pictures of Facial Affect. Palo Alto, California: Consulting Psychologists Press (1976).

46. Gray HA, Tickle-Degnen L. A meta-analysis of performance on emotion recognition tasks in Parkinson's disease. Neuropsychology (2010) 24(2):176-91. doi:10.1037/a0018104

47. Trampe D, Quoidbach J, Taquet M. Emotions in everyday life. PLoS One (2015) 10(12):e0145450. doi:10.1371/journal.pone.0145450

48. Dogan I, Sass C, Mirzazade S, Kleiman A, Werner CJ, Pohl A, et al. Neural correlates of impaired emotion processing in manifest Huntington's disease. Soc Cogn Affect Neurosci (2014) 9(5):671-80. doi:10.1093/scan/nst029

49. Pohl A, Anders S, Schulte-Ruther M, Mathiak K, Kircher T. Positive facial affect - an fMRI study on the involvement of insula and amygdala. PLoS One (2013) 8(8):e69886. doi:10.1371/journal.pone.0069886

50. Sokal RR, Rohlf FJ. Biometry: The Principles and Practice of Statistics in Biological Research. New York: W. H. Freemand and Company (1995).

51. DeCarlo LT. On the meaning and use of kurtosis. Psychol Methods (1997) 2(3):292-307. doi:10.1037//1082-989x.2.3.292 
52. Mann HB, Whitney DR. On a test of whether one of 2 random variables is stochastically larger than the other. Ann Math Stat (1947) 18(1):50-60. doi:10.1214/aoms/1177730491

53. Ashburner J, Friston KJ. Rigid body registration. 2nd ed. In: Frackowiak RS, editor. Human Brain Function. San Diego: Academic Press (2003).

54. Collins DL, Neelin P, Peters TM, Evans AC. Automatic 3D intersubject registration of MR volumetric data in standardized Talairach space. J Comput Assist Tomogr (1994) 18(2):192-205. doi:10.1097/00004728-199403000-00005

55. Evans AC, Marrett S, Neelin P, Collins L, Worsley K, Dai W, et al. Anatomical mapping of functional activation in stereotactic coordinate space. Neuroimage (1992) 1(1):43-53. doi:10.1016/1053-8119(92)90006-9

56. Ashburner J, Friston KJ. Unified segmentation. Neuroimage (2005) 26(3): 839-51. doi:10.1016/j.neuroimage.2005.02.018

57. Maldjian JA, Laurienti PJ, Kraft RA, Burdette JH. An automated method for neuroanatomic and cytoarchitectonic atlas-based interrogation of fMRI data sets. Neuroimage (2003) 19(3):1233-9. doi:10.1016/S1053-8119(03)00169-1

58. Eickhoff SB, Paus T, Caspers S, Grosbras MH, Evans AC, Zilles K, et al. Assignment of functional activations to probabilistic cytoarchitectonic areas revisited. Neuroimage (2007) 36(3):511-21. doi:10.1016/j.neuroimage. 2007.03.060

59. Eickhoff SB, Stephan KE, Mohlberg H, Grefkes C, Fink GR, Amunts K, et al. A new SPM toolbox for combining probabilistic cytoarchitectonic maps and functional imaging data. Neuroimage (2005) 25(4):1325-35. doi:10.1016/j.neuroimage.2004.12.034

60. Suzuki A, Hoshino T, Shigemasu K, Kawamura M. Disgust-specific impairment of facial expression recognition in Parkinson's disease. Brain (2006) 129:707-17. doi:10.1093/brain/awl011

61. Caspers S, Zilles K, Laird AR, Eickhoff SB. ALE meta-analysis of action observation and imitation in the human brain. Neuroimage (2010) 50(3):1148-67. doi:10.1016/j.neuroimage.2009.12.112

62. Zhen Z, Fang H, Liu J. The hierarchical brain network for face recognition. PLoS One (2013) 8(3):e59886. doi:10.1371/journal.pone.0059886

63. Wild B, Erb M, Eyb M, Bartels M, Grodd W. Why are smiles contagious? An fMRI study of the interaction between perception of facial affect and facial movements. Psychiatry Res (2003) 123(1):17-36. doi:10.1016/S0925-4927 (03)00006-4

64. Dima D, Stephan KE, Roiser JP, Friston KJ, Frangou S. Effective connectivity during processing of facial affect: evidence for multiple parallel pathways. J Neurosci (2011) 31(40):14378-85. doi:10.1523/jneurosci.2400-11.2011

65. Buccino G, Vogt S, Ritzl A, Fink GR, Zilles K, Freund HJ, et al. Neural circuits underlying imitation learning of hand actions: an event-related fMRI study. Neuron (2004) 42(2):323-34. doi:10.1016/s0896-6273(04)00181-3

66. Grezes J, Armony JL, Rowe J, Passingham RE. Activations related to "mirror" and "canonical" neurones in the human brain: an fMRI study. Neuroimage (2003) 18(4):928-37. doi:10.1016/S1053-8119(03)00042-9

67. van der Velde J, Gromann PM, Swart M, Wiersma D, de Haan L, Bruggeman R, et al. Alexithymia influences brain activation during emotion perception but not regulation. Soc Cogn Affect Neurosci (2015) 10(2):285-93. doi:10.1093/ scan/nsu056

68. Assogna F, Palmer K, Pontieri FE, Pierantozzi M, Stefani A, Gianni W, et al. Alexithymia is a non-motor symptom of Parkinson disease. Am J Geriatr Psychiatry (2012) 20(2):133-41. doi:10.1097/JGP.0b013e318209de07

69. Montgomery KJ, Haxby JV. Mirror neuron system differentially activated by facial expressions and social hand gestures: a functional magnetic resonance imaging study. J Cogn Neurosci (2008) 20(10):1866-77. doi:10.1162/ jocn.2008.20127

70. Adolphs R, Damasio H, Tranel D, Cooper G, Damasio AR. A role for somatosensory cortices in the visual recognition of emotion as revealed by three-dimensional lesion mapping. J Neurosci (2000) 20(7):2683-90.

71. Luo CY, Guo XY, Song W, Chen Q, Yang J, Gong QY, et al. The trajectory of disturbed resting-state cerebral function in Parkinson's disease at different
Hoehn and Yahr stages. Hum Brain Mapp (2015) 36(8):3104-16. doi:10.1002/ hbm. 22831

72. Tessitore A, Esposito F, Vitale C, Santangelo G, Amboni M, Russo A, et al. Default-mode network connectivity in cognitively unimpaired patients with Parkinson disease. Neurology (2012) 79(23):2226-32. doi:10.1212/WNL. 0b013e31827689d6

73. Tessitore A, Giordano A, De Micco R, Russo A, Tedeschi G. Sensorimotor connectivity in Parkinson's disease: the role of functional neuroimaging. Front Neurol (2014) 5:180. doi:10.3389/fneur.2014.00180

74. Dosenbach NUF, Fair DA, Miezin FM, Cohen AL, Wenger KK, Dosenbach RAT, et al. Distinct brain networks for adaptive and stable task control in humans. Proc Natl Acad Sci U S A (2007) 104(26):11073-8. doi:10.1073/pnas. 0704320104

75. Tunik E, Rice NJ, Hamilton A, Grafton ST. Beyond grasping: representation of action in human anterior intraparietal sulcus. Neuroimage (2007) 36:T77-86. doi:10.1016/j.neuroimage.2007.03.026

76. Dafotakis M, Sparing R, Eickhoff SB, Fink GR, Nowak DA. On the role of the ventral premotor cortex and anterior intraparietal area for predictive and reactive scaling of grip force. Brain Res (2008) 1228:73-80. doi:10.1016/j. brainres.2008.06.027

77. Rodriguez V, Thompson R, Stokes M, Brett M, Alvarez I, Valdes-Sosa M, et al. Absence of face-specific cortical activity in the complete absence of awareness: converging evidence from functional magnetic resonance imaging and event-related potentials. J Cogn Neurosci (2012) 24(2):396-415. doi:10.1162/jocn_a_00137

78. Germine LT, Garrido L, Bruce L, Hooker C. Social anhedonia is associated with neural abnormalities during face emotion processing. Neuroimage (2011) 58(3):935-45. doi:10.1016/j.neuroimage.2011.06.059

79. Nachev P, Kennard C, Husain M. Functional role of the supplementary and pre-supplementary motor areas. Nat Rev Neurosci (2008) 9(11):856-69. doi: $10.1038 / \mathrm{nrn} 2478$

80. Lehericy S, Ducros M, Krainik A, Francois C, Van de Moortele PF, Ugurbil K, et al. 3-D diffusion tensor axonal tracking shows distinct SMA and pre-SMA projections to the human striatum. Cereb Cortex (2004) 14(12):1302-9. doi:10.1093/cercor/bhh091

81. Nambu A, Takada M, Inase M, Tokuno H. Dual somatotopical representations in the primate subthalamic nucleus: evidence for ordered but reversed bodymap transformations from the primary motor cortex and the supplementary motor area. J Neurosci (1996) 16(8):2671-83.

82. Skidmore FM, Yang M, Baxter L, von Deneen KM, Collingwood J, He G, et al. Reliability analysis of the resting state can sensitively and specifically identify the presence of Parkinson disease. Neuroimage (2013) 75:249-61. doi:10.1016/j.neuroimage.2011.06.056

83. Faul F, Erdfelder E, Lang AG, Buchner A. G*Power 3: a flexible statistical power analysis program for the social, behavioral, and biomedical sciences. Behav Res Methods (2007) 39(2):175-91. doi:10.3758/Bf03193146

84. Iacoboni M, Dapretto $M$. The mirror neuron system and the consequences of its dysfunction. Nat Rev Neurosci (2006) 7(12):942-51. doi:10.1038/ nrn2024

Conflict of Interest Statement: The authors declare that the research was conducted in the absence of any commercial or financial relationships that could be construed as a potential conflict of interest.

Copyright (c) 2017 Pohl, Anders, Chen, Patel, Heller, Reetz, Mathiak and Binkofski. This is an open-access article distributed under the terms of the Creative Commons Attribution License (CC BY). The use, distribution or reproduction in other forums is permitted, provided the original author(s) or licensor are credited and that the original publication in this journal is cited, in accordance with accepted academic practice. No use, distribution or reproduction is permitted which does not comply with these terms. 